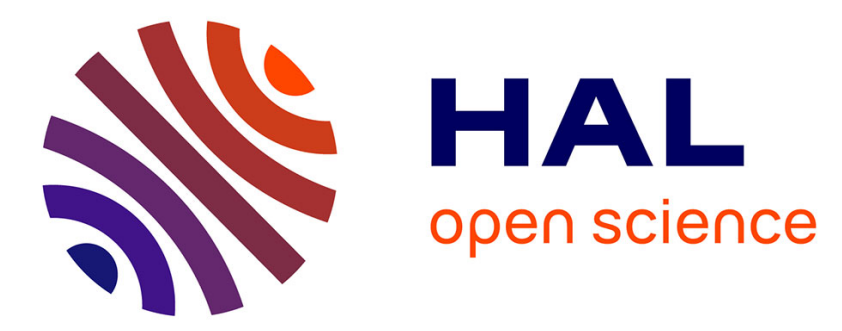

\title{
Utilisation des DEL et photodiodes PIN pour la transmission haute qualité de signaux TV par fibre optique
}

\author{
Murielle Richard, P. Roux
}

\section{- To cite this version:}

Murielle Richard, P. Roux. Utilisation des DEL et photodiodes PIN pour la transmission haute qualité de signaux TV par fibre optique. Revue de Physique Appliquée, 1984, 19 (2), pp.95-97. 10.1051/rphysap:0198400190209500 . jpa-00245179

\section{HAL Id: jpa-00245179 https://hal.science/jpa-00245179}

Submitted on 1 Jan 1984

HAL is a multi-disciplinary open access archive for the deposit and dissemination of scientific research documents, whether they are published or not. The documents may come from teaching and research institutions in France or abroad, or from public or private research centers.
L'archive ouverte pluridisciplinaire HAL, est destinée au dépôt et à la diffusion de documents scientifiques de niveau recherche, publiés ou non, émanant des établissements d'enseignement et de recherche français ou étrangers, des laboratoires publics ou privés. 


\title{
Utilisation des DEL et photodiodes PIN pour la transmission haute qualité de signaux TV par fibre optique
}

\author{
M. Richard et P. Roux \\ CLTO, 35, rue Jean-Jaurès, BP 20, 95871 Bezons Cedex, France
}

(Reçu le 3 juin 1983, révisé le 26 septembre, accepté le 19 octobre 1983)

\begin{abstract}
Résumé. - L'utilisation de diodes électroluminescentes et de photodiodes PIN pour la transmission TV en bande de base par fibres optiques nécessite certaines précautions d'emploi, eu égard aux phénomènes de distorsions et d'échauffement inhérents à ces composants. Après avoir indiqué les remèdes apportés lors de la réalisation d'une liaison opérationnelle, nous en donnerons les principales caractéristiques obtenues sur le terrain.
\end{abstract}

\begin{abstract}
The use of LEDs and PIN photodiodes for optical TV base-band transmission requires special care to reduce distorsions and thermal problems which are relevant to these components. We present the different solutions we investigated, and we give the characteristics obtained in an operationnal optical link.
\end{abstract}

\section{Introduction.}

La majorité des réalisations et des publications traitant de la transmission TV par fibre optique est consacrée à l'introduction de modulations intermédiaires plus ou moins faciles à mettre en œuvre (FM, PFM, numérique...) et à l'utilisation de composants de haut de gamme comme la diode laser et la photodiode PDA. Ce faisant, on s'éloigne des applications de distribution pour lesquelles un faible coût de l'ensemble de la liaison est recherché.

Cet exposé décrit la réalisation de terminaux optoélectroniques permettant la transmission de qualité de signaux TV sur plusieurs kilomètres de fibres optiques, tout en utilisant les solutions élémentaires comme la transmission en bande de base et les composants opto-électroniques les plus accessibles : diode électroluminescente et photodiode PIN fonctionnant autour de $850 \mathrm{~nm}$. Nous détaillons les principaux éléments constitutifs d'une telle liaison, en indiquant les limitations à en attendre et les principales caractéristiques.

\section{Eléments constitutifs.}

Comme tous les systèmes de transmission par fibre optique, cette liaison peut se décomposer en trois sous-ensembles :

- L'émetteur optique, constitué de la diode électroluminescente GaAlAs et de son circuit de modulation;
- La ligne optique (fibre et câble);

- Le récepteur optique comprenant la photodiode PIN silicium suivie de ses circuits d'amplification et de remise en forme.

\section{Emetteur optique (cf. Fig. 1).}

La carte émission optique comporte à son entrée un étage d'adaptation, permettant de fonctionner avec des composantes continues différentes $(0-1 \mathrm{~V}$ à $-500 \mathrm{mV}$, $+500 \mathrm{mV}$ ). Après une amplification en tension réglable en fonction de l'affaiblissement de la ligne et de la qualité requise pour la liaison - puis en courant, on dispose de $200 \mathrm{~mA}$, nécessaire pour un taux de modulation élevé, sous $6 \mathrm{~V}$ pour moduler la diode électroluminescente ( $2 \mathrm{~V}$ seulement étant nécessaires pour la diode elle-même).

L'utilisation de diodes électroluminescentes entraîne deux types de distorsions du signal [1] :

- L'échauffement du composant, dû à des densités de courants de commande relativement élevés, se traduit par une dérive thermique et une décroissance de la puissance optique en présence de paliers lors d'un "signal au blanc ». Cet effet introduit une non-linéarité BF qu'on peut compenser, en partie, par une sélection rigoureuse du composant (il semble que ce défaut soit plus important avec les diodes à double hétérostructure) et une modulation la plus réduite possible.

- Les niveaux de courant importants appliqués 


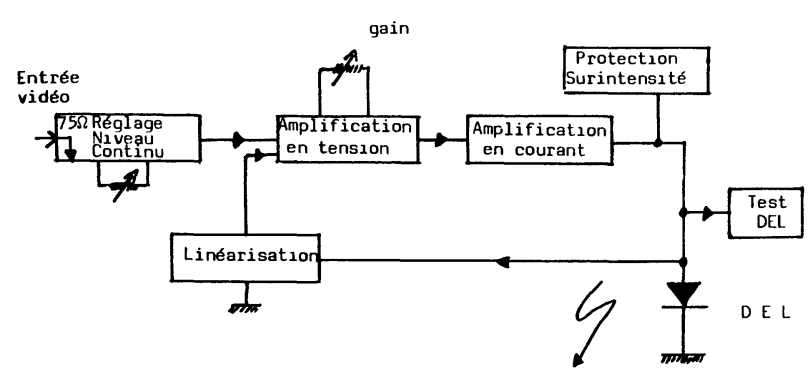

Fig. 1. - Schéma synoptique de l'émission.

[Block diagram of the transmitter.]

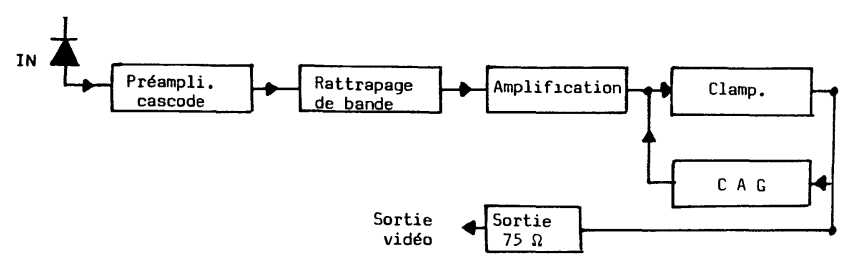

Fig. 2. - Schéma synoptique de la réception.

[Block diagram of the receiver.]

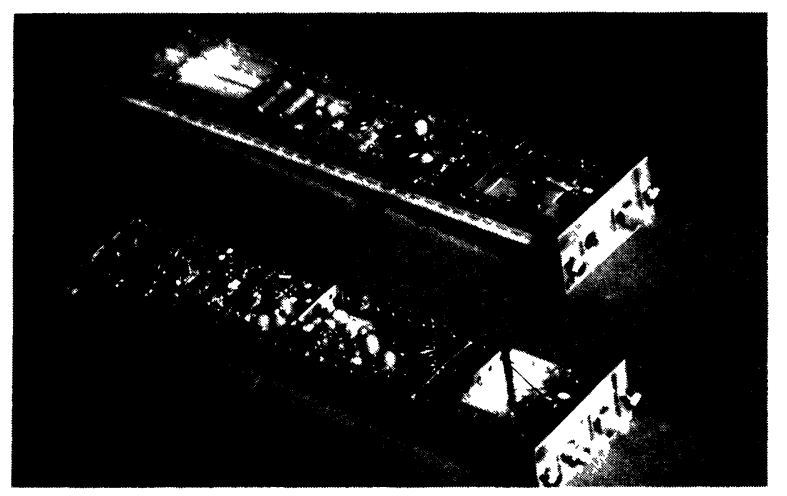

à la diode produisent des distorsions introduites par les non-linéarités de la caractéristique de transfert (puissance en fonction du courant) [2]. Afin d'en limiter les conséquences, et en particulier d'améliorer le gain différentiel, la linéarité et la phase différentielle, on effectue une prédistorsion du signal en limitant l'intensité, par l'intermédiaire d'une boucle de contreréaction.

Pour cela, on introduit dans cette boucle un réseau non linéaire à base de trois diodes qui deviennent successivement conductrices lorsque leur seuil respectif est atteint. On obtient une modification par paliers du gain et ainsi du courant de commande du composant optique. Il faut cependant veiller à régler unitairement ces seuils dans la mesure où toutes les diodes électroluminescentes ne présentent pas une courbe de transfert courant-puissance optique identique. Comme il est très délicat de visualiser l'effet de correction de nonlinéarité au niveau du composant opto-électronique seul (sinon en accouplant une photodiode), les réglages se font une fois la liaison terminée, en optimisant les paramètres à l'aide d'un oscilloscope.
De plus, un écrêteur d'intensité, placé à la sortie de l'étage d'amplification, évite une détérioration accidentelle de la diode d'émission. Le niveau d'écrêtage peut être ajusté en fonction du type de diode utilisé (certaines diodes tolérant des courants directs voisins de $200 \mathrm{~mA}$ en régime continu).

Un autre problème fondamental au niveau de l'émission optique est la transmission, sans altération, des tops de synchronisation dont le niveau — fixé théoriquement à $300 \mathrm{mV}$ - influe sur l'amplitude du signal de sortie en réception. Or, la caractéristique de transfert de la DEL n'étant pas linéaire et la valeur moyenne d'un signal vidéo n'étant pas constante, le niveau bas des tops de synchronisation varie. Il faut donc fixer les limites de modulation et, pour cela, transmettre la composante continue.

\section{Récepteur optique (cf. Fig. 2).}

Le signal optique est détecté par une photodiode PIN, choisie pour son faible temps de montée (qq. ns) et sa bonnè sensibilité $(0,5 \mathrm{~A} / \mathrm{W})$.

Le niveau de réception étant très faible, une préamplification à faible bruit est nécessaire. Nous avons choisi un préamplificateur de type cascode car ce montage, à haute impédance d'entrée, a l'avantage reconnu d'une grande sensibilité. Toutefois, sa bande passante relativement faible nécessite qu'un étage d'égalisation suive celui de préamplification.

Un gain en tension, appliqué au signal à l'aide de trois transistors montés en émetteur commun, permet de restituer les niveaux nécessaires. Il faut noter que, eu égard aux problèmes d'échauffement, le couplage de ces étages se fait par liaison capacitive.

Nous avons expliqué, précédemment, que la hauteur du bas des tops de synchronisation est ramenée à une tension fixée par un système à diodes. En réception, un dispositif de «clamp " aligne le niveau haut de ces tops de synchronisation et le compare aux $300 \mathrm{mV}$ qui devraient être obtenus si le gain de la liaison était de 1 . Cette mesure agit sur un étage de commande automatique de gain (CAG) qui compense alors le manque ou l'excès de gain. La dynamique de cette régulation est faible car il est très délicat de combiner une CAG importante d'une part, et, d'autre part, un temps de réponse minimum.

\section{Résultats obtenus.}

En insérant entre cet émetteur et ce récepteur optique un câble de trois kilomètres, comportant une fibre $50 / 125$ d'atténuation inférieure à $3 \mathrm{~dB} / \mathrm{km}$, à $850 \mathrm{~nm}$, les résultats obtenus sur le terrain sont les suivants [3] :

- Bande passante : $10 \mathrm{MHz}$ à - $3 \mathrm{~dB}$.

- Linéarité : $<3 \%$.

- Gain différentiel : $<3 \%$.

- Phase différentielle $:<4,5^{\circ}$.

- Rapport S/B pondéré (image à $100 \%$ de blanc) : $>61 \mathrm{~dB}$ 
- Dynamique de réception : $5 \mathrm{~dB}$ optique.

- Sensibilité du récepteur optique : $-30 \mathrm{dBm}$ pour un rapport S/B de $60 \mathrm{~dB}$.

- Puissance couplée à l'émission, dans une fibre de type 50/125:- $20 \mathrm{dBm}$.
Ils montrent, notamment en ce qui concerne le rapport signal à bruit, une compatibilité avec la qualité requise pour les lignes de transfert telles qu'on peut les envisager pour les futurs réseaux locaux, qu'ils soient publics ou privés.

\section{Bibliographie}

[1] Asatani, K. and Kimura, T., Non linear distorsions of L.E.Ds., IOOC 77.

[2] Ayre, R., Linearity of Led for Analogue Optical Fibre Links, ATR 1973.

[3] Lесомте, J. et al., Réalisation d'une ligne de transfert en fibre optique, Revue Radiodiffusion Télévision 78 juin-août 1983. 